\title{
Genome-scale CRISPR screening for potential targets of ginsenoside compound $\mathrm{K}$
}

\author{
Yuanyuan Yang ${ }^{1}$, Xiaojian Liu', Shuang Li', Yanhao Chen'1, Yongxu Zhao', Yuda Wei', Yan Qiu', Yan Liu', \\ Zhihua Zhou', Jun $\mathrm{Han}^{3}$, Guohao Wu $\mathbb{1 0}^{3}$ and Qiurong Ding (1)
}

\begin{abstract}
Ginsenosides exhibit a large variety of biological activities in maintaining physical health; however, the molecule underpinnings underlining these biological activities remain to be defined. Here, we took a cellular condition that compound K (CK) induces autophagic cell death in HeLa cells, and setup a high-throughput genetic screening using CRISPR technology. We have identified a number of CK-resistant and CK-sensitive genes, and further validated PMAIP1 as a CK-resistant gene and WASH1 as a CK-sensitive gene. Compound $\mathrm{K}$ treatment reduces the expression of WASH1, which further accelerates the autophagic cell death, highlighting WASH1 as an interesting downstream mediator of CK effects. Overall, our study offers an easy-to-adopt platform to study the functional mediators of ginsenosides, and provides a candidate list of genes that are potential targets of CK.
\end{abstract}

\section{Introduction}

Ginseng presents a valuable traditional herbal medicine that has been widely used in Asia for millenniums to maintain physical healthy. Its active constituents are ginsenosides, a group of triterpene sapoins, which exhibit a large variety of biological activities, such as anti-tumor ${ }^{1}$, anti-inflammatory ${ }^{2}$, anti-aging ${ }^{3}$, etc. Of more than onehundred kinds of ginsenosides, compound $\mathrm{K}$ (20-O-betaD-glucopyranosyl-20(S)-protopanaxadiaol, CK) is a major deglycosylated metabolite form, which is absorbed and discovered in circulation ${ }^{4}$. The diverse biological effects of CK is well explored and recognized, and with recent success in constructing "yeast factories" that can produce large amount of CK with high purity ${ }^{5}$, CK presents a

\footnotetext{
Correspondence: Jun Han (hanjun198626@163.com) or

Guohao Wu (profwugh@163.com) or Qiurong Ding (qrding@sibs.ac.cn)

${ }^{1}$ CAS Key Laboratory of Nutrition, Metabolism and Food Safety, Shanghai Institute of Nutrition and Health, Shanghai Institutes for Biological Sciences, University of Chinese Academy of Sciences, Chinese Academy of Sciences, Shanghai 200031, P. R. China

${ }^{2}$ CAS-Key Laboratory of Synthetic Biology, Institute of Plant Physiology and Ecology, Shanghai Institutes for Biological Sciences, Chinese Academy of Sciences, Shanghai 200032, China

Full list of author information is available at the end of the article. These authors contributed equally: Yuanyuan Yang, Xiaojian Liu Edited by G. M. Fimia
}

promising potential therapeutic agent for many diseases $^{3,6-8}$.

Many signaling pathways and molecular targets have been reported to mediate the diverse biological functions of CK. In tumor cells, CK exhibits a direct cytotoxic and growth-inhibitory effects via pathways including induction of caspase-dependent apoptosis and cell cycle arrest ${ }^{9}$, and increase of mitochondrial disruption. In atherosclerosis and neurodegenerative ${ }^{10}$ conditions, CK treatment can have anti-inflammation and anti-allergic effects, with molecular mechanisms mostly centered around inhibitory functions to NF- $\kappa B^{7,11,12}$ and MAPK pathways $^{13}$, ROS generation ${ }^{9}$, NADPH oxidase activity ${ }^{5}$, and production of inflammatory cytokines $^{14}$. Besides, depending on different cell types studied, many other targets have also been identified, e.g. $\mathrm{AMPK}^{4}$, calcium flux ${ }^{15}$, altogether constituting a complicated signaling network that can be influenced by CK treatment.

Despite of all these efforts, there has been no systematic and unbiased analysis of potential downstream targets of CK so far. With recent advances in genome editing technologies, especially the clustered regularly interspaced short palindromic repeats (CRISPR)/CRISPRassociated (Cas) system, genome-wide genetic screening

\section{(c) The Author(s) 2020}

(c) Open Access This article is licensed under a Creative Commons Attribution 4.0 International License, which permits use, sharing, adaptation, distribution and reproduction cc) in any medium or format, as long as you give appropriate credit to the original author(s) and the source, provide a link to the Creative Commons license, and indicate if changes were made. The images or other third party material in this article are included in the article's Creative Commons license, unless indicated otherwise in a credit line to the material. If material is not included in the article's Creative Commons license and your intended use is not permitted by statutory regulation or exceeds the permitted use, you will need to obtain permission directly from the copyright holder. To view a copy of this license, visit http://creativecommons.org/licenses/by/4.0/. 
using CRISPR technologies offers a high-throughput, lowcost, and unbiased platform that enables comprehensive study of genetic regulators in a biological condition ${ }^{16}$. We thus wanted to employ this genetic screening approach to identify potential downstream targets of CK. To enable a successful genetic screening, we took advantage of a cellular condition that CK treatment can promote autophagic cell death, which allows a cell-survival-based readout for screening ${ }^{17}$. We then applied genome-wide CRISPR screening technology to this cell model. We have identified a number of "CK-resistant genes"-loss of which can render cells more resistant to CK treatment; and "CK-sensitive genes"-loss of which can make cells more sensitive to CK treatment, offering a useful resource for further exploring the molecular underpinnings to various biological activities of CK. Our study also demonstrates a broadly useful approach for studying the potential molecular targets of traditional herbal medicines.

\section{Materials and methods Cell culture}

Human embryonic kidney $293 \mathrm{~T}$ cells, human cervix adenocarcinoma HeLa cells, and human hepatocarcinoma HuH-7 cells were purchased from Cell Bank (SIBS, Shanghai), and maintained in DMEM (Dulbecco's modified Eagle's medium) supplemented with $10 \%$ fetal bovine serum and $1 \%$ penicillin/streptomycin. Cells were grown in a humidified $\mathrm{CO}_{2}$ incubator at $37^{\circ} \mathrm{C}$.

For chemical treatment, HeLa cells and $\mathrm{HuH} 7$ cells were either cultured in a serum-free medium and treated with compound $\mathrm{K}$ ( $5 \mathrm{nM}$ ) (A gift form Dr. Zhihua Zhou) for different times as indicated; or cultured in full medium and treated with compound $\mathrm{K}(5 \mathrm{nM}, 5 \mu \mathrm{M}$, or $15 \mu \mathrm{M})$ for different days as indicated. 3-Methyladenine (3-MA, MedChemExpress, 5142-23-4, $5 \mathrm{mM}$ ) was applied 1 day before compound $\mathrm{K}$ treatment.

\section{Lentivirus packaging}

To prepare lentiviruses for CRISPR library or a single CRISPR plasmid, HEK293T cells in each $10 \mathrm{~cm}$ dish were transfected with $22.5 \mu \mathrm{g}$ CRISPR plasmids together with $14.7 \mu \mathrm{g}$ pMDL, $5.7 \mu \mathrm{g}$ pRev, and $7.9 \mu \mathrm{g}$ pVSVG packaging plasmids. After transfection, medium with viral particles were collected $48 \mathrm{~h}$ and $72 \mathrm{~h}$ later and centrifuged at 20,000 r.p.m at $4{ }^{\circ} \mathrm{C}$ for $2 \mathrm{~h}$ to pellet viral particles. Viral pellets were then resuspended in DMEM at $4{ }^{\circ} \mathrm{C}$ overnight and titer was calculated using a PCR-based titration kit (Applied Biological Materials Inc, LV900).

\section{Genome-wide CRISPR-Cas9 screenings}

The genome-wide CRISPR library was purchased from Addgene (\#1000000048). Library was amplified following the protocol provided by Addgene, and lentiviruses were prepared and titer was calculated as described above.

For genetic screening, around $3.4 \times 10^{7} \mathrm{HeLa}$ cells were infected by the CRISPR lentivirus library to achieve an infection efficiency at around $30 \%$. After virus infection, cells were treated with puromycin $(1 \mu \mathrm{g} / \mathrm{ml})$ for 1 day to eliminate noninfected cells. Cells were then treated with DMSO as control, or compound $\mathrm{K}(5 \mathrm{nM})$ for 2 days, allowed to recover for 1 day without drug treatment, and treated with compound $\mathrm{K}$ for one more day before harvesting.

Genomic DNA of cells from different groups (DMSO control group and compound $\mathrm{K}$ treatment group) was extracted and the sgRNAs were amplified by two-rounds PCR method using KOD DNA polymerase (TOYOBO, KOD-401). In the first round, a total of $2.2 \mu \mathrm{g}$ genomic DNA (200 ng per PCR reaction; 11 separate reactions for each sample) from each group was used as DNA template; the PCR program used was $94{ }^{\circ} \mathrm{C} 5 \mathrm{~min}, 98^{\circ} \mathrm{C} 30 \mathrm{~s}$, $57^{\circ} \mathrm{C} 30 \mathrm{~s}, 68^{\circ} \mathrm{C} 35 \mathrm{~s}, 18$ cycles. The primers were as following: $5^{\prime}$ - TGAAAGTATTTCGATTTCTTG GCTT $-3^{\prime}$ and 5'-CGGTGCCACTTTTTCAAGTT-3'. The products (288 bp) were applied in the second round of PCR as template, the PCR program used was $94{ }^{\circ} \mathrm{C}$ $5 \mathrm{~min}, 98^{\circ} \mathrm{C} 30 \mathrm{~s}, 57^{\circ} \mathrm{C} 30 \mathrm{~s}, 68^{\circ} \mathrm{C} 35 \mathrm{~s}, 24$ cycles. PCR primers used for amplification were as following: $5^{\prime}$ Barcode + TGAAAGTATTTCGATTTCTTGGCTT-3', and $\quad 5^{\prime}$-Barcode + CGGTGCCACTTTTTCAAGTT- $3^{\prime}$. An 8 bp barcode for multiplexing of different biological samples were added at $5^{\prime}$ of each primer. Products (142 bp) were gel-purified and quantified. In total $3.6 \mu \mathrm{g}$ PCR products from each group were pooled together and subjected for deep sequencing (Illumina HiSeq4000 system).

For data analysis, raw reads of sgRNA were demultiplexed using the FASTX-Toolkit (http://hannonlab.cshl. edu/fastx_toolkit/) and processed to contain only the unique sgRNA sequence. The sgRNA sequences from the GeCKO library were then assembled into a BurrowsWheeler index using the Bowtie ${ }^{18}$ build-index function. The sgRNA information from deep sequencing were further aligned to the index using the Bowtie 2 aligner. After alignment, the read count statistics for each library sequence was preform using $\mathrm{MAGeCK}^{19}$. Significant genes in both positive selection analysis and negative selection analysis are simultaneously identified based on the sgRNA statistical significance of each gene using MAGeCK, using FDR $<0.05$.

\section{Construction of the RGLC3 reporter}

The mRFP-GFP-LC3 fragment was constructed using a PCR-based ligation method, which was then inserted between the XbaI and BamHI sites of pLentiCRISPR V2 (Addgene, \#52961) to replace the original Cas9 cassette, 
to obtain the lentivirus-mRFP-GFP-LC3 (Lenti-RGLC3) construct.

Lenti-RGLC3 was then packaged into lentivirus, and used to infect HeLa cells to monitor autophagic influx. The fluorescence was imaged using a fluorescence microscope (Olympus BX53).

\section{Quantitative RT-PCR analysis}

Total RNA was extracted from HeLa using TRIzol reagent (ThermoFisher, 15,596,018) and transcribed with the reverse transcription kit (Takara, RR047A). Quantitative real-time PCR was carried out on the 7900 System (ABI) using SYBR Green supermix (ABI, 4472908). The sequences of primers were as following: $5^{\prime}$-CGGCTAC CACATCCAAGGAA- $3^{\prime}$ and $5^{\prime}$ - GCTGGAATTACCGC GGCT-3' for human 18S RNA; 5'-AACGACCTCATG TACAGTGC-3' and 5'-GTGTTAGTACCCCATCTTGT AGG-3' for human WASH1 gene. 5'-ACCAAGCCGGA TTTGCGATT- $3^{\prime}$ and $5^{\prime}$ - ACTTGCACTTGTTCCTCG TGG $-3^{\prime}$ for human PMAIP1 gene.

\section{Generation of CRISPR-Cas9 knockout cell lines}

The WASH1-KO and PMAIP1-KO cell lines were generated by the CRISPR/Cas9 technology. The $20 \mathrm{bp}$ sequences of sgRNA targeting individual genes was inserted into plentiCRISPR V2 and used for lentivirus packaging. The target sequences used are $5^{\prime}$-CAGG CACCATGACTCCTGTG-3' for human WASH1, and 5'TCGAGTGTGCTACTCAACTC-3' for human PMAIP1. Lentiviruses carrying CRISPR-Cas9 targeting individual genes or empty plentiCRISPR V2 as control viruses were packaged. HeLa cells were then infected and selected with puromycin to remove uninfected cells. Cells were next subjected to genomic DNA extraction for T7EI analysis (NEB, E3321) or protein extraction for western blot to determine gene editing efficiency. Primers used in T7EI analysis for each gene were as follows: 5' - AAATCAA TGGTTGTGCACGGTT-3' and 5'-TAGCAAGCACCTT GTAGGGG-3' for human WASH1;5'-GGACAAAAGC GTGGTCTCTGGCG-3' and 5' - CTCCTGAACACAGG GGCCCTTG-3' for human PMAIP1.

\section{Generation of WASH1 overexpressing cell line}

For exogenous overexpression of WASH1 in HeLa cells, human WASH1 cDNA was amplified, and inserted to the pCDH-EF1 vector (System Biosciences, CD520A1) between the XbaI and NotI sites, to obtain the pCDHWASH1 construct. Primers used to amplify WASH1 cDNA were as following: 5'- GCTCTAGAATGACTC CTGTGAGGATGCA $-3^{\prime}$ and 5'-ACGAGGACGACT GGGAATCGGCGGCCGCTAAACTAT-3'. The pCDHWASH1 construct was then packaged into lentivirus, and used to infect HeLa cells for exogenous overexpression.

\section{Transmission electron microscopy imaging}

HeLa cells were fixed overnight with $2.5 \%$ glutaraldehyde and $2 \%$ paraformaldehyde in cacodylate buffer $(0.1 \mathrm{M}, \mathrm{pH}$ 7.4). The ultrathin sections were obtained on an ultra cryomicrotome (Ultra Microtome Reichert Ultracut E; Leica Microsystems, Wetzlar, Germany) and were visualized with Joel JEM-1230 transmission electron microscope (TEM).

\section{Hoechst 33258 staining assay}

Hoechst 33258 (ThermoFisher, H3569) staining was performed to capture apoptotic induction of CK to HeLa cells. HeLa cells cultured in serum-free medium were treated with CK $(5 \mathrm{nM})$ or DMSO for 1 or 2 days, before fixed with $4 \%$ paraformaldehyde for $30 \mathrm{~min}$ at $4{ }^{\circ} \mathrm{C}$. Cells were then stained with Hoechst 33258 solution for $10 \mathrm{~min}$ at room temperature and subjected to imaging using a fluorescence microscope (Olympus BX53).

\section{Flow cytometry assay}

HeLa cells cultured in serum-free medium were treated with CK $(5 \mathrm{nM})$ or DMSO for 1 day. Cells and supernatant were then collected and centrifuged, with the cell pellet resuspended in $195 \mu \mathrm{L}$ binding buffer (Beyotime, C1062S). Cells were later stained with the FITC-Annexin V apoptosis detection kit (Beyotime, C1062S) according to manufacturer's instructions, and analyzed by flow cytometry using the CytoFLEX S (BECKMAN COULTER).

\section{Western blot analysis}

Protein from cells was extracted by RIPA buffer (Millipore, 20,188) and subjected to regular western procedure. The primary antibodies used in the experiments were alpha-tubulin (Sigma, T6557), $\beta$-Actin (CST, 8H10D10), LC3B (Sigma, ABC432), WASH C1 (Sigma, HPA002689), PMAIP1(ABclonal, A9801)

\section{Statistical analysis}

The unpaired, two-tailed Student's $t$-test was used for experiments with two groups and one-way ANOVA with post-hoc Bonferroni multiple-comparison tests were used for experiments containing more than two groups. All data are represented as means with SEM. ImageJ software was used for quantification of image signals.

\section{Results}

\section{Compound $\mathrm{K}$ treatment induces autophagic cell death}

CK treatment to tumor or nontumor cells can have a direct toxic and growth-inhibitory effect ${ }^{20-24}$, offering an appropriate cellular condition that can be applied in the high-throughput genetic screening via a cell-survivalbased readout. We first evaluated the effects of $\mathrm{CK}$ treatment to HeLa cell line, which is a cervical tumor cell 


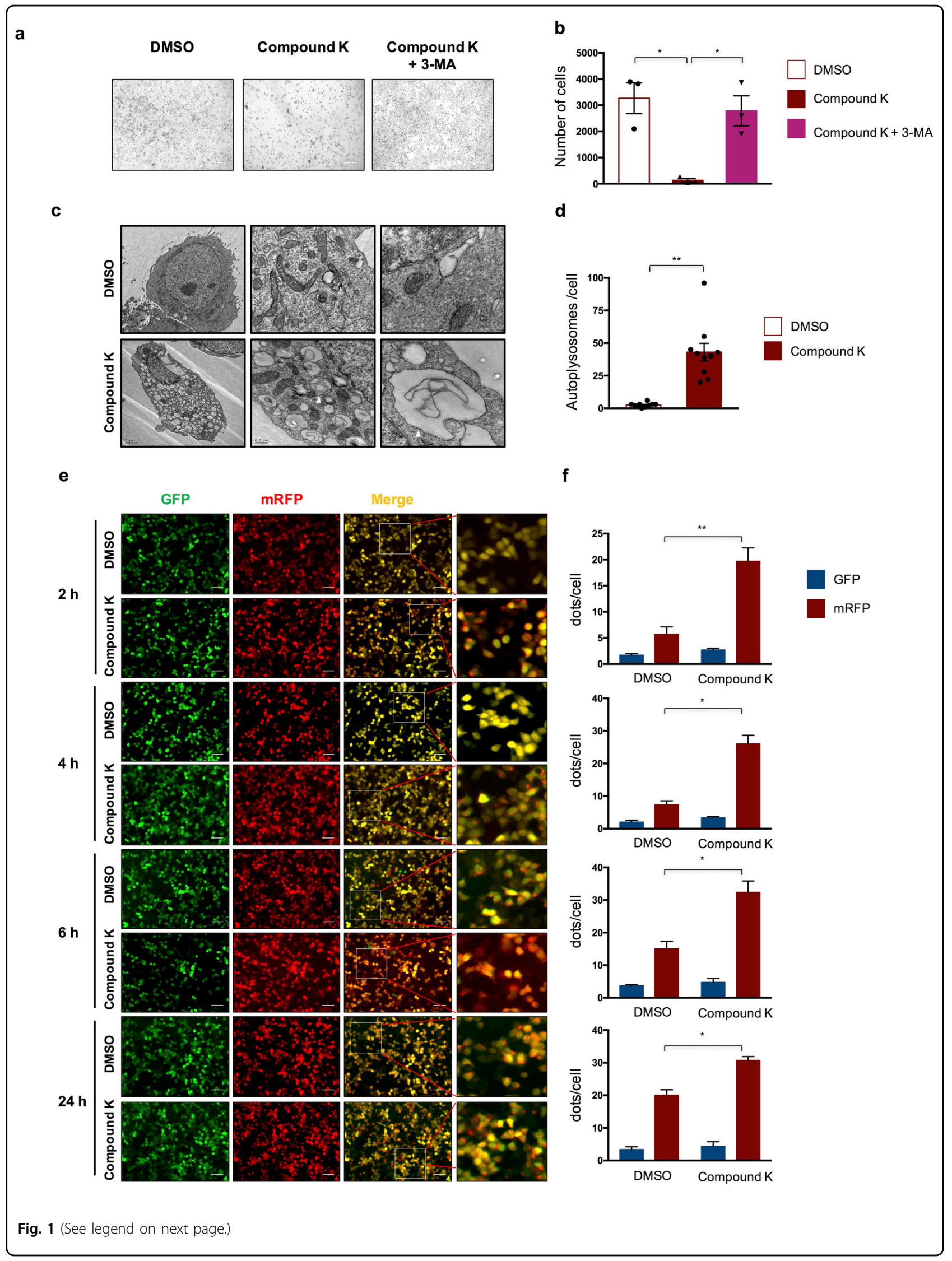


(see figure on previous page)

Fig. 1 Compound $\mathbf{K}$ treatment induces autophagic cell death in HeLa cells. a Representative images of cell state after CK (5 nM) or DMSO treatment for 3 days, with or without pretreatment with 3-MA (5 mM) for 1 day. Scale bar $=150 \mu \mathrm{m}$. b Quantification of cell numbers in each cellular condition as presented in a. c Representative EM images of cells after CK (5 nM) or DMSO treatment. Scale bar $=2 \mu \mathrm{m}$ (left), $0.5 \mu \mathrm{m}$ (middle), $0.2 \mu \mathrm{m}$ (right). Arrow head, autolysomes. d Quantification of autolysosomes in each cellular condition as presented in c. e Representative images of cellular localization of RGLC3 probe after CK (5 nm) or DMSO treatment for different times as indicated. Scale bar $=150 \mu \mathrm{m}$. $\mathbf{f}$ Quantification of average dots per cell of mRFP and GFP signals in each cellular condition as presented in e. Data are represented as means with SEM. ${ }^{*} P<0.05$, ${ }^{* *} P<0.01$.

line, and has been widely used in tumor studies. CK treatment at a low concentration $(5 \mathrm{nM})$ for 3 days in a FBS starvation medium induced a massive cell death (Fig. 1a, b, Fig. S1a, b); whereas the cell death can be reversed by pretreating the cells with 3-methyladenine (3-MA), which is a selective inhibitor to class III phosphatidylinositol 3-kinase (PtdIns3K) and can also block the formation of autophagosome ${ }^{25}$ (Fig. 1a, b). The remaining cells after $\mathrm{CK}$ treatment also displayed significantly more autolysosomes, as compared to control cells, under the transmission electron microscope (TEM) (Fig. 1c, d). Altogether, these results suggest autophagic cell death induced by CK treatment.

We further confirmed this phenomenon by using the fluorescent probe, GFP-mRFP-LC3, to evaluate autophagic flux ${ }^{26}$. The membrane-bound microtubule associated protein 1 light chain 3 pro (LC3) is fused with green and red fluorescent proteins. GFP, but not mRFP, is unstable in acidic environment, and undergoes degradation in autolysomes. The GFP/mRFP ratio can therefore be calculated to estimate autophagic flux. HeLa cells were infected by lentivirus-mRFP-GFP-LC3 (Lenti-RGLC3), and then was treated with DMSO or CK for $1-24 \mathrm{~h}$. Consistent with previous findings, the induction of autophagy was more obvious and the autophagic flux was significantly accelerated in CK-treatment groups, as compared to control cells (Fig. 1e, f). Further tests in full medium containing FBS also demonstrated the induction of autophagy and cell death upon CK treatment, albeit at a much higher concentration (Fig. S1c, d). Additionally, similar phenomenon was observed in $\mathrm{HuH7}$ cells after CK treatment (Fig. S2). Taken together, these results indicate that CK treatment induces autophagic cell death in HeLa as well as HuH7 cells.

\section{High-throughput screenings identify potential CK-sensitive and CK-resistant genes}

To further explore the potential targets of CK, we setup a genome-wide genetic screening by using the GeCKO library ${ }^{16}$. GeCKO library lentiviruses were applied to infect HeLa cells at an efficiency of around 30\%, to ensure that most of the cells were infected by one virus. Cells were then selected with puromycin to remove uninfected cells, before further treatment with CK or DMSO control. Cells infected with control virus were also treated with $\mathrm{CK}$ and used as a negative control (Fig. 2a). Consistent with previous results, $\mathrm{CK}$ treatment induced a massive cell death. However, there are significantly more cells left in GeCKO virus-infected cells, as compared to cells infected with control viruses (Fig. 2b). The survival cells in GeCKO group were then grown up, and genomic DNA was further isolated for deep sequencing to retrieve the gRNA information. Two independent screenings were performed, and cells infected with GeCKO library and treated with DMSO control were included for gRNA diversity comparison.

We did both positive selection analysis and negative selection analysis. Positive selection analysis aims to identify gRNAs that are more enriched in survival cells after CK treatment, as compared to the DMSO control group, which enables the discovery of CK-resistant genes - loss of which can render cells more resistant to CKinduced cell death. Whereas negative selection analysis is intended to identify gRNAs that are more depleted in survival cells after CK treatment, emphasizing the discovery of CK-sensitive genes-loss of which can make cells more sensitive to CK-induced cell death. In both analyses, using FDR $<0.05$ as a cutoff, we have identified 584 CK-resistant genes in positive selection analysis (Table. S1), and 368 CK-sensitive genes in negative selection analysis (Table. S2).

In CK-resistant genes, we noticed that several of the top genes are functionally related to cell growth and cell survival (Fig. 2c). For example, PMAIP1, encoding phorbal-12-myristate-13-acetate-induced protein 1, promotes activation of caspases and apoptosis; ${ }^{27}$ KIAA0141, also called DELE1, encoding DAP3-binding cell death enhancer 1 , is essential for the induction of death receptormediated apoptosis through regulation of caspase activation $^{28}$. Analysis of the 584 CK-resistant genes revealed significant enrichment in pathways in negative regulation of cell population proliferation (41 genes), positive regulation of cell communication (69 genes), regulation of MAPK cascade (36 genes), etc (Fig. 2d).

In CK-sensitive genes, several miRNAs stood out in the top genes (Fig. 2e). More interestingly, analysis of the 368 CK-sensitive genes revealed enriched pathways related to RNA process, including mRNA splicing, mRNA catabolic, RNA localization, mRNA export from nucleus (Fig. 2f). The molecular underpinnings behind this phenomenon are worth further investigation. 
a
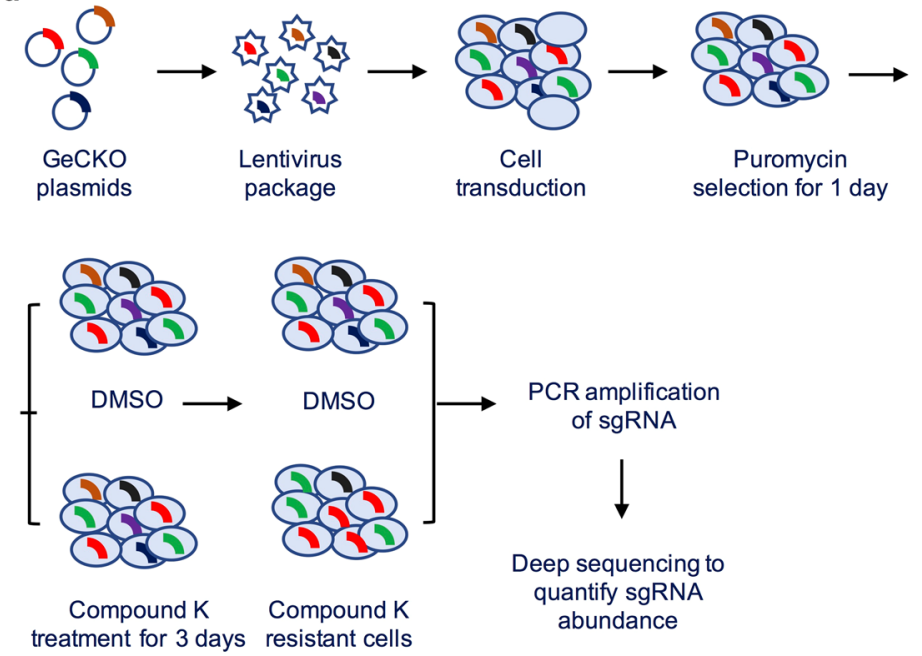

d

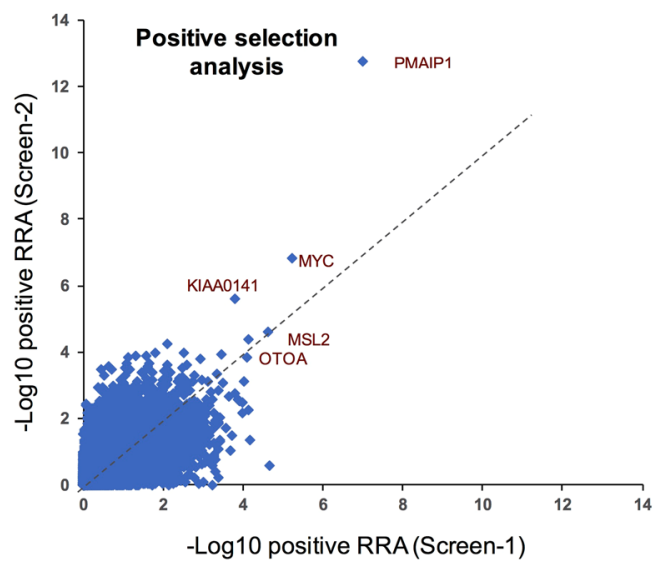

b
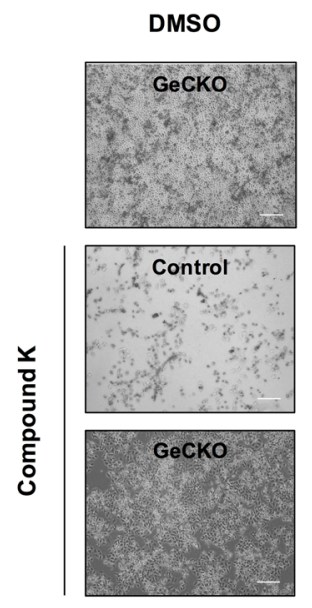
treatment for 3 days resistant cells

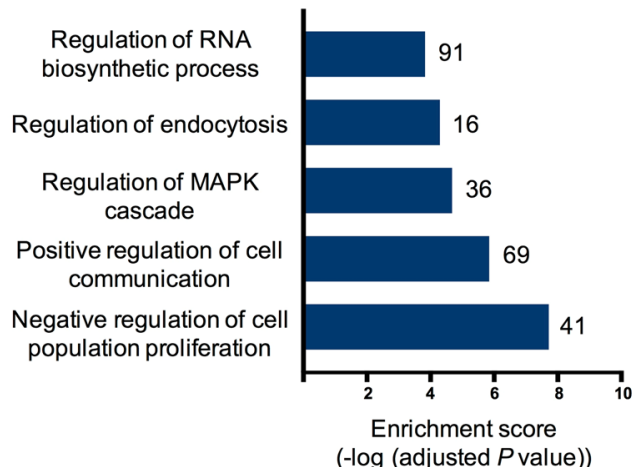

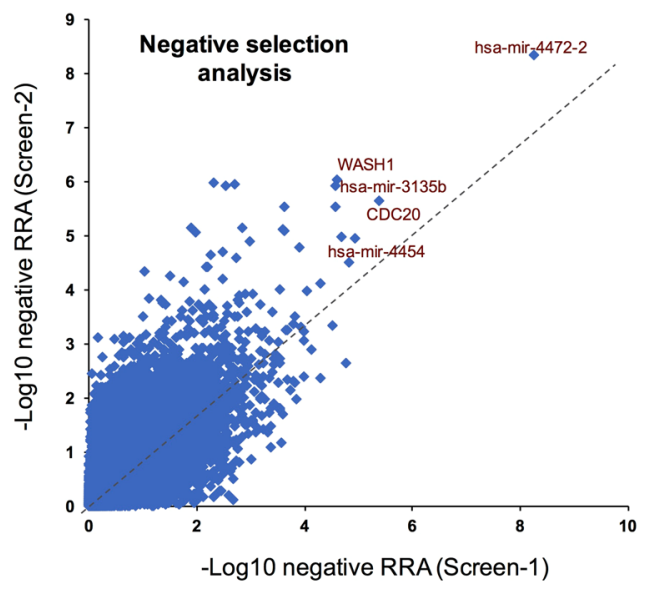

f

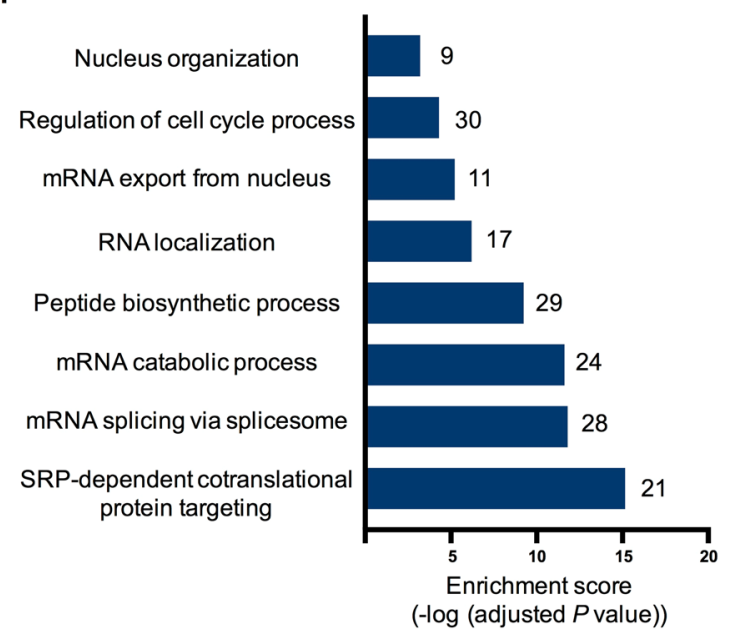


(see figure on previous page)

Fig. 2 High-throughput screenings identify CK-resistant and CK-sensitive genes. a Schematic view of the experimental setup in the highthroughput genetic screenings. b Representative images of cell state at each treatment condition as indicated. Scale bar $=150 \mu \mathrm{m}$. c Scatterplot showing significant genes in positive selection analysis. $\mathbf{d}$ Gene Oncology and pathway analysis of positive selection genes. e Scatterplot showing significant genes in negative selection analysis. $\mathbf{f}$ Gene Oncology and pathway analysis of negative selection genes. RRA, Robust Ranking Aggregation score generated in the MAGeCK algorithm.

a

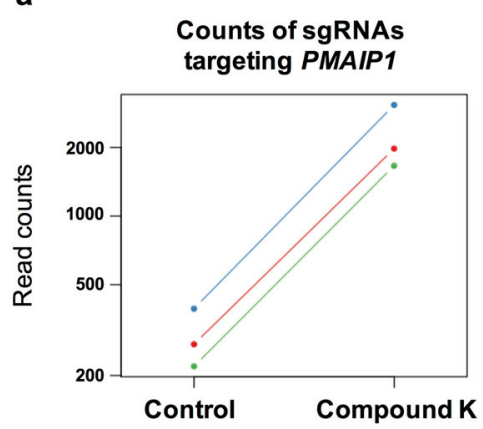

C

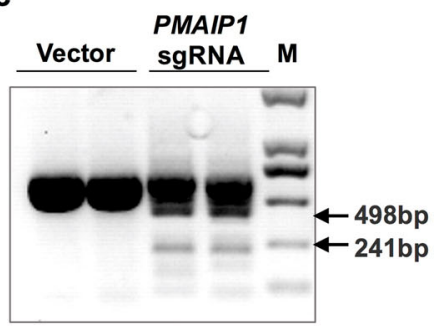

f

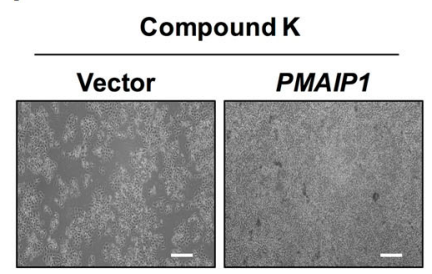

b

PMAIP1

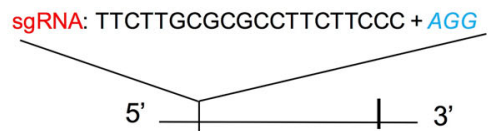

d

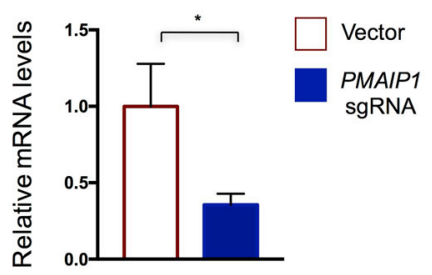

e

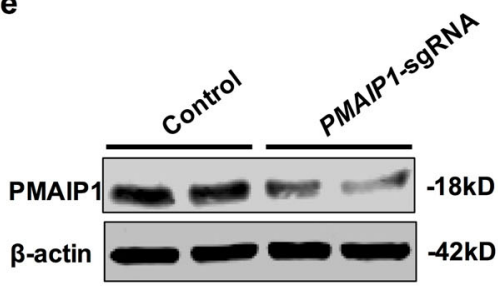

h

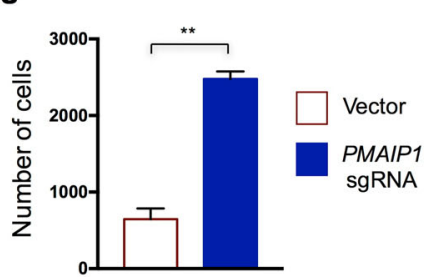

Compound $\mathrm{K}$

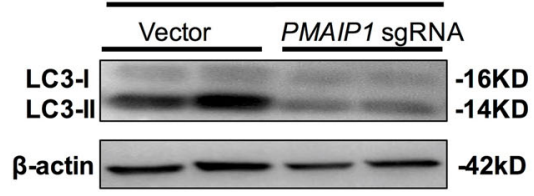

Fig. 3 PMAIP1 is a CK-resistant gene. a Read counts of sgRNAs targeting PMAIP1 in control and CK-treated groups. $\mathbf{b}$ Illustration of the sgRNA applied to deplete PMAIP1 in validation experiments. c Genome editing activity as assessed by T7E1 assay of sgRNA targeting PMAIP1. d Relative mRNA expression level of PMAIP1 in control and PMAIP1 sgRNA-treated cells. e Analysis of the protein level of PMAIP1 in control and PMAIP1 sgRNAtreated cells. $\mathbf{f}$ Representative images of cell state after CK (5 nM) treatment for 3 days. Scale bar $=150 \mu \mathrm{m}$. $\mathbf{g}$ Quantification of cell numbers in each cellular condition as presented in $\mathbf{f}$. $\mathbf{h}$ Analysis of the LC3 protein level in control and PMAIP1 sgRNA-treated cells after CK treatment for $1 \mathrm{~h}$. Data are represented as means with SEM. ${ }^{*} P<0.05,{ }^{* *} P<0.01$.

\section{PMAIP1 knockout cells are resistant to autophagic cell death induced by compound $\mathrm{K}$ treatment}

We further did validation of these top hits in both analyses. PMAIP1, also named NOXA, is a top hit in positive selection analysis. All three gRNAs targeting PMAIP1 displayed a significant enrichment in survival cells after CK treatment (Fig. 3a). PMAIP1 encodes a $\mathrm{BH} 3$-containing mitochondrial protein, which can disrupt mitochondrial outer membrane integrity and cause the apoptosis $^{29}$. To further validate the functional involvement of PMAIP1 in cell death caused by CK treatment, we simply targeted PMAIP1 via CRISPR-Cas9 technology in HeLa cells (Fig. 3b). CRISPR targeting resulted in a clear cutting at the PMAIP1 genomic locus as revealed by the T7 endonuclease 1 (T7E1) assay (Fig. 3c), and subsequently significant reduction in mRNA expression due to nonsense mediated decay (Fig. 3d), and protein expression (Fig. 3e). Consistent with the screening result, PMAIP1-targeted cells showed significant resistance in cell death in response to CK treatment (Fig. 3f, g). 
a

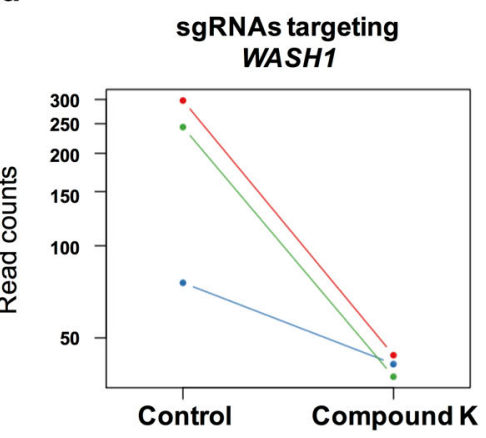

b

WASH1

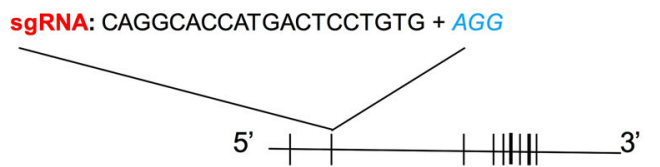

e
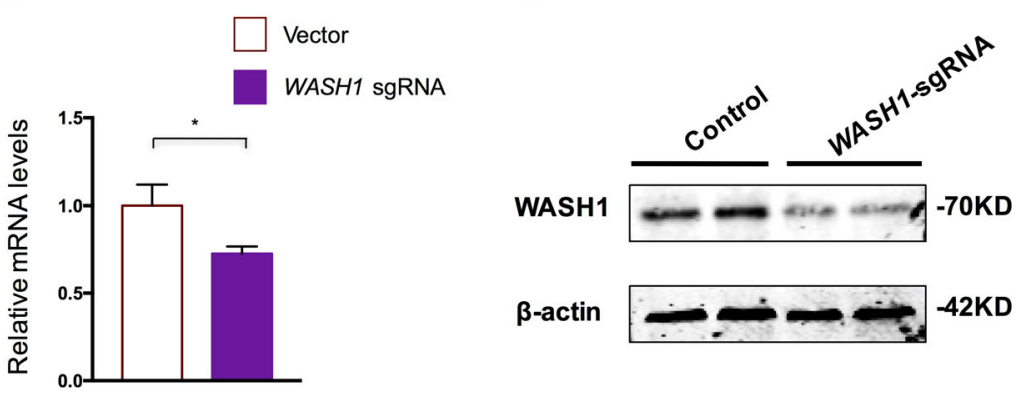

f
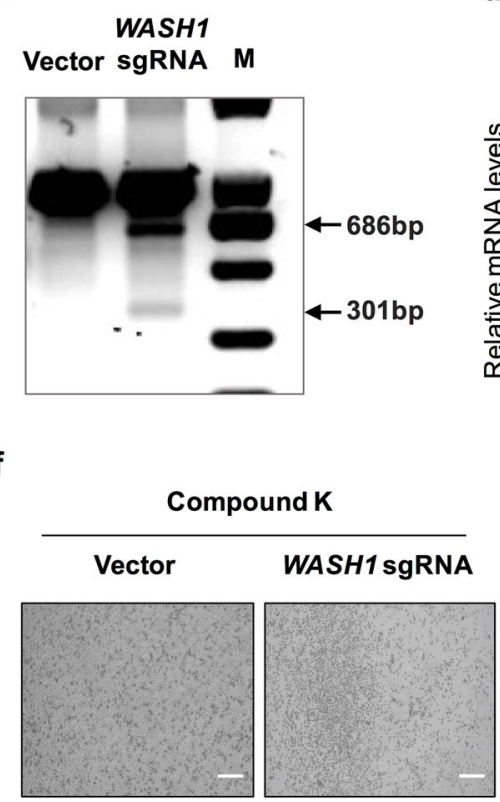

g

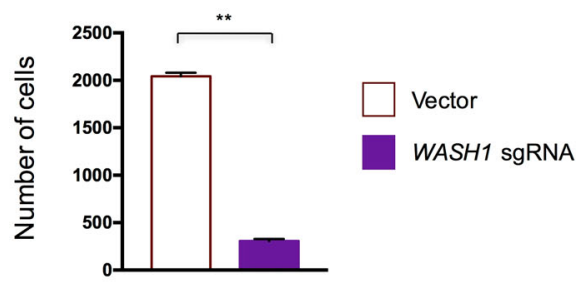

Fig. 4 WASH1 is a CK-sensitive gene. a Read counts of sgRNAs targeting WASH1 in control and CK-treated groups. $\mathbf{b}$ Illustration of the sgRNA applied to deplete WASH1 in validation experiments. c Genome editing activity as assessed by T7E1 assay of sgRNA targeting WASH1. d Relative mRNA expression level of WASH1 in control and WASH1 sgRNA-treated cells. e Analysis of the protein level of WASH1 in control and WASH1 sgRNAtreated cells. $\mathbf{f}$ Representative images of cell state after CK $(5 \mathrm{nM})$ treatment for 1 day. Scale bar $=150 \mu \mathrm{m}$. $\mathbf{g}$ Quantification of cell numbers in each cellular condition as presented in $\mathbf{f}$. Data are represented as means with SEM. ${ }^{*} P<0.05,{ }^{* *} P<0.01$.

Additionally, further analysis demonstrated a reduced autophagy induction process in PMAIP1-targeted cells, as revealed by less lipidated LC3 (Fig. 3h). However, CK treatment did not alter the expression of PMAIP1 at mRNA or protein level (Fig. S3).

\section{WASH1 knockout cells are more sensitive to autophagic cell death induced by compound $\mathrm{K}$ treatment}

We next focused on one of top hits in negative selection analysis. WASH1, encoding WASH complex subunit 1, which is previously known to function during endosome sorting and endocytic trafficking ${ }^{30-32}$, and also in regulation of autophagy independently from its role in endosomal sorting ${ }^{33}$. All three gRNAs targeting WASH1 displayed a consistent depletion in survival cells after CK treatment, ranking WASH1 as a significant negative selection gene (Fig. 4a). To further validate the role of WASH1 in CK-induced cell death, CRISPR technology was adopted to target WASH1 in HeLa cells (Fig. 4b). CRISPR targeting led to an obvious cutting at the WASH1 genomic locus as revealed by the T7 endonuclease 1 (T7E1) assay (Fig. 4c), resulting in significant decrease in mRNA level of WASH1 (Fig. 4d), and elimination of major WASH1 proteins (Fig. 4e). Importantly, when WASH1targeted cells were subjected to CK treatment, these cells displayed a hypersensitive reaction to $\mathrm{CK}$, with dying of the majority of cells after only 1-day treatment (Fig. 4f, g).

Further analysis demonstrated a stronger autophagy induction process in WASH1-targeted cells, as revealed by more lipidated LC3 (Fig. 5a, b), accelerated autophagic 


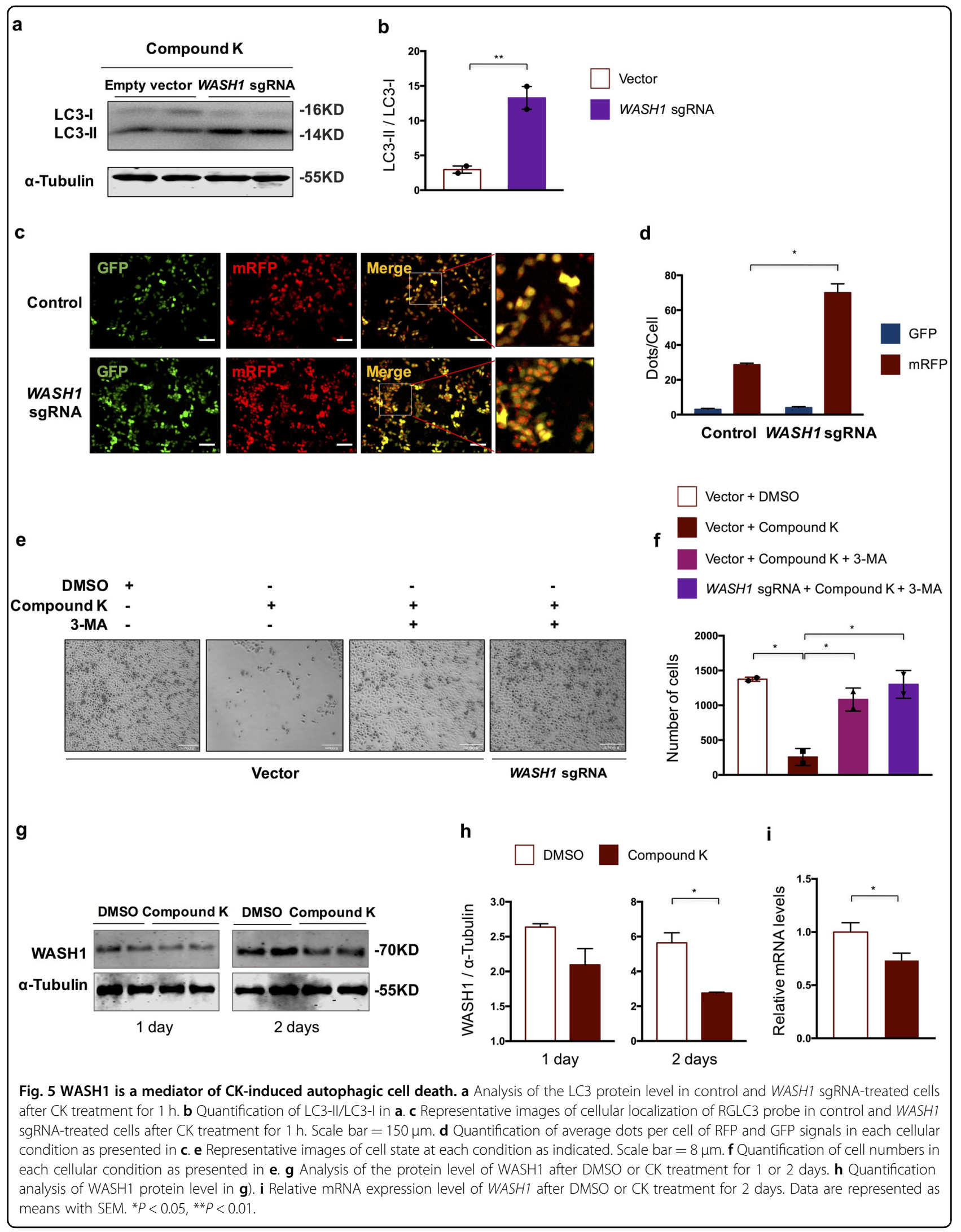


influx (Fig. 5c, d), in comparison to control cells, and the reverse of cell death by pretreatment with 3-MA (Fig. 5e, f). These results altogether demonstrate that WASH1 is a negative regulator in CK-induced autophagic cell death.

We next examined whether CK treatment affects WASH1 expression in HeLa cells. Indeed, we found decreased WASH1 expression in HeLa cells upon CK treatment, both at the protein level (Fig. $5 \mathrm{~g}, \mathrm{~h}$ ) and at the mRNA level (Fig. 5i). These results indicated that CK treatment led to decreased cellular WASH1 expression, which releases the negative control of autophagy and leads to autophagic cell death.

To examine whether recovery WASH1 expression is enough to rescue the CK-induced autophagic cell death, we increased WASH1 expression in cells via exogenous lentiviral expression (Fig. S4a, b). However, we did not observe significant protective effect after WASH1 overexpression (Fig. S4c, d), suggesting that other than the WASH1 pathway, there are also other pathways involved in CK-induced cell death.

\section{Discussion}

Given the promising therapeutic potentiality of compound $\mathrm{K}$ as well as other ginsenosides ${ }^{1-3}$, it remains an intriguing question regarding the specific molecules and pathways that mediate the variety effects of a certain ginsenoside. Here, we addressed this question by performing a CRISPR-based high-throughput genetic screening in CK-treated HeLa cells, and identified a number of CK-resistant and CK-sensitive genes. We further validated PMAIP1 as a CK-resistant gene and WASH1 as a CK-sensitive gene, pointing out WASH1 as an interesting downstream mediator of $\mathrm{CK}$, which is involved in CK-induced autophagic cell death.

In this study, we took advantage of a cellular condition that CK treatment induces autophagic cell death, offering a cell-survival-based readout for screening. While cell survival as a readout is often adopted and favorable in setting up high-throughput genetic screenings, this presents an extreme cellular condition, thus cannot represent many other functional effects of CK treatment. In the positive selection analysis, a number of candidate genes are involved in regulation of cell growth or apoptosis ${ }^{28,34-36}$, which is in fact common in a cell survival based screening, and cannot being simply considered to be specific to CK treatment. However, there are also many other genes that are not functionally connected to cell survival that have being identified in the screening. It is interesting to see that genes enriched in the regulation of endocytosis and RNA biosynthetic process have been identified in positive selections, and genes enriched in many aspects of RNA processing and even nucleus organization have been identified in the negative selection. In fact, in the further validation experiments, we have confirmed WASH1 as a
CK-sensitive gene. CK treatment reduces the expression of WASH1 at both the transcriptional and translational levels, which further accelerates the autophagic cell death, with WASH-1 depleted cells becoming hypersensitive to $\mathrm{CK}$ treatment. These results are consistent with the previous discovery that WASH1 is a negative regulator of autophagy. However, WASH1 is also an important regulator in endocytosis ${ }^{30-32}$, whether CK treatment can also affect cell endocytic trafficking and cargo sorting via WASH1 warrants further investigation. The presentation of RNA processing related genes in both positive and negative selection analyses, suggest that CK treatment may also affect RNA biology directly or indirectly, raising an interesting direction for further functional pursuing of ginsenosides.

Taken together, our study, for the first time, has established a high-throughput genetic screening, by which a number of CK-resistant and CK-sensitive genes have been identified. We have also confirmed the involvement of two genes, PMAIP1 and WASH1, in CK treatment induced autophagic cell death in HeLa cells. Many other targets will also need to be further validated before conclusions can be made. Nonetheless, our study reveals interesting targets of compound $\mathrm{K}$, and offers one easy-toadopt platform to study the potential functional mediators of ginsenosides.

\section{Acknowledgements}

This work was supported by grants from the National Key R\&D Program of China (2017YFA0102800, 2017YFA0103700, 2018YFA0900700), the Strategic Priority Research Program of the Chinese Academy of Sciences (XDA16030402), the National Natural Science Foundation of China (31670829, 31971063), and China Postdoctoral Science Foundation (2019M661661).

\section{Author details \\ ${ }^{1}$ CAS Key Laboratory of Nutrition, Metabolism and Food Safety, Shanghai Institute of Nutrition and Health, Shanghai Institutes for Biological Sciences, University of Chinese Academy of Sciences, Chinese Academy of Sciences, Shanghai 200031, P. R. China. ${ }^{2}$ CAS-Key Laboratory of Synthetic Biology, Institute of Plant Physiology and Ecology, Shanghai Institutes for Biological Sciences, Chinese Academy of Sciences, Shanghai 200032, China. ${ }^{3}$ Department of General Surgery, Zhongshan Hospital, Fudan University, Shanghai, China. ${ }^{4}$ Institute for Stem Cell and Regeneration, Chinese Academy of Sciences, Beijing 100101, P. R. China}

\section{Author contributions}

J.H., G.W., and Q.D. designed the research. Y.Y. performed most of the experiments and. X.L. did GeCKO analysis. S.L., Y.C., Y.Z., Y. W., Y.Q, Y. L., Y.W. and Z.Z. assisted with either experiments or data analysis. Y.Y., X.L., and Q.D. wrote the manuscript. J.H., G.W., and Q.D. supervised the project.

Conflict of interest

The authors declare that they have no conflict of interest.

\section{Publisher's note}

Springer Nature remains neutral with regard to jurisdictional claims in published maps and institutional affiliations.

Supplementary Information accompanies this paper at (https://doi.org/ 10.1038/s41419-020-2234-5) 
Received: 17 September 2019 Revised: 3 January 2020 Accepted: 7 January 2020

Published online: 20 January 2020

\section{References}

1. Kim, Y. J. et al. Development of Lactobacillus kimchicus DCY51(T)-mediated gold nanoparticles for delivery of ginsenoside compound $\mathrm{K}$ : in vitro photothermal effects and apoptosis detection in cancer cells. Artif. Cells Nanomed. Biotechnol. 47, 30-44 (2019).

2. Lee, J. O. et al. Compound $K$, a ginsenoside metabolite, plays an antiinflammatory role in macrophages by targeting the AKT1-mediated signaling pathway. J. Ginseng Res. 43, 154-160 (2019).

3. Yang, X. D., Yang, Y. Y., Ouyang, D. S. \& Yang, G. P. A review of biotransformation and pharmacology of ginsenoside compound K. Fitoterapia 100, 208-220 (2015).

4. Li, C. et al. Ginsenoside metabolite compound $\mathrm{K}$ induces apoptosis and autophagy in non-small cell lung cancer cells via AMPK-mTOR and JNK pathways. Biochem. Cell Biol. 97, 406-414 (2019).

5. Yan, $\mathrm{X}$. et al. Production of bioactive ginsenoside compound $\mathrm{K}$ in metabolically engineered yeast. Cell Res. 24, 770-773 (2014).

6. Seo, J. Y., Ju, S. H., Oh, J., Lee, S. K. \& Kim, J. S. Neuroprotective and cognitionenhancing effects of compound $\mathrm{K}$ isolated from red ginseng. J. Agric Food Chem. 64, 2855-2864 (2016).

7. Song, W., Wei, L., Du, Y., Wang, Y. \& Jiang, S. Protective effect of ginsenoside metabolite compound $\mathrm{K}$ against diabetic nephropathy by inhibiting NLRP3 inflammasome activation and NF-kappaB/p38 signaling pathway in high-fat diet/streptozotocin-induced diabetic mice. Int. Immunopharmacol. 63 , 227-238 (2018).

8. Zhang, $X$. et al. Compound $K$ induces endoplasmic reticulum stress and apoptosis in human liver cancer cells by regulating STAT3. Molecules https:// doi.org/10.3390/molecules23061482 (2018).

9. Oh, J. M., Kim, E. \& Chun, S. Ginsenoside compound K induces Ros-mediated apoptosis and autophagic inhibition in human neuroblastoma cells in vitro and in vivo. Int. J. Mol. Sci. https://doi.org/10.3390/ijms20174279 (2019).

10. Oh, J. \& Kim, J. S. Compound K derived from ginseng: neuroprotection and cognitive improvement. Food Funct. 7, 4506-4515 (2016).

11. Zhang, J. et al. Ginsenosides regulate PXR/NF-kappaB signaling and attenuate dextran sulfate sodium-induced colitis. Drug Metab. Dispos. 43, 1181-1189 (2015).

12. Li, J. et al. Ginsenoside metabolite compound K promotes recovery of dextran sulfate sodium-induced colitis and inhibits inflammatory responses by suppressing NF-kappaB activation. PLoS ONE 9, e87810 (2014).

13. Lu, S. et al. Ginsenoside compound K protects human umbilical vein endothelial cells against oxidized low-density lipoprotein-induced injury via inhibition of nuclear factor-kappaB, p38, and JNK MAPK pathways. J. Ginseng Res. 43, 95-104 (2019).

14. Chen, J. et al. Ginsenoside metabolite compound K exerts anti-inflammatory and analgesic effects via downregulating COX2. Inflammopharmacology 27, 157-166 (2019).

15. Kim, K., Park, M., Lee, Y. M., Rhyu, M. R. \& Kim, H. Y. Ginsenoside metabolite compound $\mathrm{K}$ stimulates glucagon-like peptide-1 secretion in $\mathrm{NCl}-\mathrm{H} 716$ cells via bile acid receptor activation. Arch. Pharm. Res. 37, 1193-1200 (2014).
16. Shalem, O., Sanjana, N. E. \& Zhang, F. High-throughput functional genomics using CRISPR-Cas9. Nat. Rev. Genet. 16, 299-311 (2015).

17. Chen, L. et al. Ginsenoside compound $K$ sensitizes human colon cancer cells to TRAIL-induced apoptosis via autophagy-dependent and -independent DR5 upregulation. Cell Death Dis. 7, e2334 (2016).

18. Langmead, B. \& Salzberg, S. L. Fast gapped-read alignment with Bowtie 2. Nat Methods 9, 357-359 (2012).

19. Li, W. et al. MAGeCK enables robust identification of essential genes from genome-scale CRISPR/Cas9 knockout screens. Genome Biol. 15, 554 (2014).

20. Zhang, M. et al. Ginsenoside compound-K inhibits the activity of B cells through inducing IgD-B cell receptor endocytosis in mice with collageninduced arthritis. Inflammopharmacology 27, 845-856 (2019).

21. Fan, $H$. Y. et al. Ginsenoside compound $K$ ameliorates imiquimodinduced psoriasis like dermatitis through inhibiting REG3A/Reglll gamma expression in keratinocytes. Biochem. Biophys. Res. Commun. 515, 665-671 (2019).

22. Kang, K. A. et al. Compound K, a metabolite of ginseng saponin, inhibits colorectal cancer cell growth and induces apoptosis through inhibition of histone deacetylase activity. Int. J. Oncol. 43, 1907-1914 (2013).

23. Law, K. M. et al. Ginsenoside compound $\mathrm{K}$ induces apoptosis in nasopharyngeal carcinoma cells via activation of apoptosis-inducing factor. Chin Med. $\mathbf{9}$, 11 (2014). https://doi.org/10.1186/1749-8546-9-11.

24. Zheng, Z. Z. et al. Compound K-induced apoptosis of human hepatocellular carcinoma MHCC97-H cells in vitro. Oncol. Rep. 32, 325-331 (2014).

25. Valcifahmetoglu-Norberg, H., Xia, H. G. \& Yuan, J. Y. Pharmacologic agents targeting autophagy. J. Clin. Investig. 125, 5-13 (2015).

26. Runwal, G. et al. LC3-positive structures are prominent in autophagy-deficient cells. Sci. Rep. 9, 10147 (2019).

27. Zhao, X., Liu, X. \& Su, L. Parthenolide induces apoptosis via TNFRSF10B and PMAIP1 pathways in human lung cancer cells. J. Exp. Clin. Cancer Res. 33, 3 (2014).

28. Wazir, U. et al. Effects of the knockdown of death-associated protein 3 expression on cell adhesion, growth and migration in breast cancer cells. Oncol. Rep. 33, 2575-2582 (2015).

29. Nguyen, D. T. et al. Mitochondrial targeting domain of NOXA causes necrosis in apoptosis-resistant tumor cells. Amino Acids 50, 1707-1717 (2018).

30. Gomez, T. S. \& Billadeau, D. D. A FAM21-containing WASH complex regulates retromer-dependent sorting. Dev. Cell 17, 699-711 (2009).

31. Jia, D. et al. WASH and WAVE actin regulators of the Wiskott-Aldrich syndrome protein (WASP) family are controlled by analogous structurally related complexes. Proc. Natl Acad. Sci. USA 107, 10442-10447 (2010).

32. Hao, Y. H. et al. Regulation of WASH-dependent actin polymerization and protein trafficking by ubiquitination. Cell 152, 1051-1064 (2013).

33. Zavodszky, E. et al. Mutation in VPS35 associated with Parkinson's disease impairs WASH complex association and inhibits autophagy. Nat. Commun. 5, 3828 (2014).

34. Bretones, G., Delgado, M. D. \& Leon, J. Myc and cell cycle control. Biochim. Biophys. Acta 1849, 506-516 (2015).

35. Xie, Y. P. et al. CDC20 regulates cardiac hypertrophy via targeting LC3dependent autophagy. Theranostics 8, 5995-6007 (2018).

36. Kruse, J. P. \& Gu, W. MSL2 promotes Mdm2-independent cytoplasmic localization of p53. J. Biol. Chem. 284, 3250-3263 (2009). 